\title{
Epidemiology, Diagnosis, and Management of Cystic Lesions of the Pancreas
}

\author{
Koen de Jong, ${ }^{1}$ Marco J. Bruno, ${ }^{2}$ and Paul Fockens ${ }^{1}$ \\ ${ }^{1}$ Department of Gastroenterology and Hepatology, Academic Medical Center, University of Amsterdam, P.O. Box 22700, \\ 1100 DE Amsterdam, The Netherlands \\ ${ }^{2}$ Department of Gastroenterology and Hepatology, Erasmus Medical Center, 3000 CA, Rotterdam, The Netherlands
}

Correspondence should be addressed to Paul Fockens, p.fockens@amc.nl

Received 2 June 2011; Accepted 25 July 2011

Academic Editor: Jose G. de la Mora-Levy

Copyright ( $) 2012$ Koen de Jong et al. This is an open access article distributed under the Creative Commons Attribution License, which permits unrestricted use, distribution, and reproduction in any medium, provided the original work is properly cited.

\begin{abstract}
Although little is known on the true prevalence of pancreatic cysts, physicians are currently more frequently confronted with pancreatic cysts because of the increasing use of sophisticated cross-sectional abdominal imaging. Cystic lesions of the pancreas comprise of a heterogeneous group of diagnostic entities, some of which are benign such as inflammatory pseudocysts or serous cystadenomas and do not require resection when asymptomatic. Others like mucinous cysts or intraductal papillary mucinous neoplasms (IPMN) have a malignant potential and in these cases surgical resection is often indicated. For this reason an adequate distinction between the various cysts is crucial to optimize management strategy. Different diagnostic methods that could be of value in the differentiation include radiologic imaging techniques such as CT, MR, and endosonography. In addition, fluid aspiration for cytopathology, tumormarkers or molecular analysis is widely used. Different guidelines are available but so far no optimal diagnostic algorithm exists. We summarize the epidemiology, classification, clinical presentation, diagnostics, management, and future perspectives.
\end{abstract}

\section{Introduction}

As a result of the widespread use of cross-sectional imaging, clinicians are confronted with pancreatic cysts with increasing frequency [1]. The majority of these cysts are asymptomatic, and the decision whether or not to operate is not always straightforward. Although our knowledge of the pathophysiology and pathobiology of pancreatic cysts is increasing, relatively little is known about their natural history.

The apparent question is how to proceed after the detection of an asymptomatic pancreatic cyst choosing one of the following options: no further investigations, additional imaging \pm fine needle aspiration (FNA), surveillance, or surgical/endoscopic treatment. Despite a spectacular improvement in diagnostic modalities in the past decades, differential diagnosis and hence management of pancreatic cysts remain controversial. Most centers have adopted a differential approach with follow up in case of absence of secondary features of malignancy and surgical resection in case of a high suspicion of malignancy. Multiple guidelines have appeared.
In this paper we will attempt to provide a comprehensive overview of the epidemiology, diagnostic options, and management of pancreatic cysts.

\section{Epidemiology}

To date only a few studies have been performed investigating the true prevalence of pancreatic cysts. We have recently published a study in which 2803 magnetic resonance imaging (MRI) examinations were retrospectively reviewed in a group of mostly asymptomatic patients who decided to undergo a preventive screening abdominal MRI at their own initiative and costs without referral of a physician. Prevalence was $2.4 \%$ and increased with age [1]. A study by Laffan et al. reported a prevalence of $2.6 \%$ [2]. In retrospect, 2832 consecutive computed tomography (CT) scans were reviewed. Patients with known pancreatic disease or symptoms related to the pancreas were excluded. A prevalence of $13.5 \%$ was found in another recent retrospective study in 616 patients 


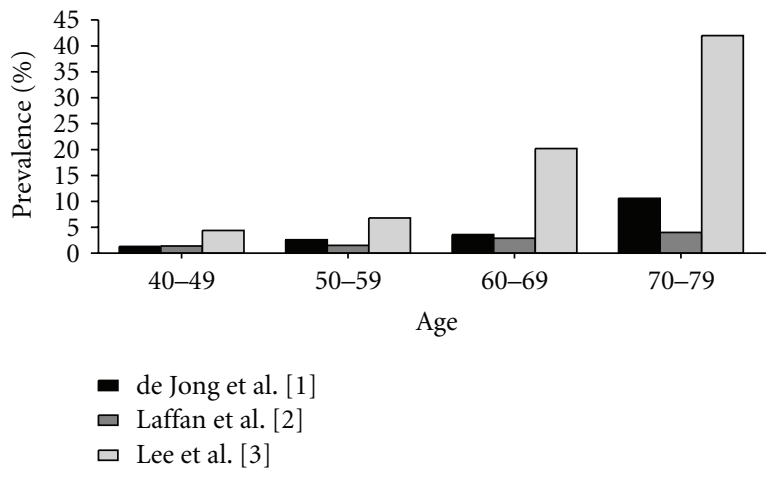

Figure 1: Prevalence of pancreatic cysts in relation to increasing age.

using MRI [3]. Patients were excluded from this study if they had a known or suspected history of pancreatic disease. In all these studies increasing age correlated with a higher prevalence of pancreatic cysts (Figure 1).

In an older Italian study reports of 24,039 MRI and CT scans were retrospectively reviewed with a computerized search. Pancreatic cysts were reported in $1.2 \%$ of which $58 \%$ ( $0.7 \%$ of total study population) did not have a history of pancreatitis [4]. The highest prevalence of pancreatic cysts using a radiologic imaging technique was found in a study by Zhang et al. [5]. Spin-echo MR images of 1444 patients were reviewed for pancreatic cysts by two radiologists, and pancreatic cysts were described in $19.6 \%$ of patients. Patients with known history of pancreatic disease were not excluded from this study.

In an autopsy study of 300 cases a stunning $24.3 \%$ were found to have pancreatic cysts [6]. It is of note that this study was performed in elderly patients (more than $80 \%$ were older than 65 years), and no information was provided of a possible history of pancreatic disease. The results of the described studies are summarized in Table 1 . The broad range of prevalence values can be explained by the fact that studies differed in the selection of the study population, in-hospital or out-patient based and whether patients with potential pancreatic disease were excluded from analysis. Importantly, studies also differed in which imaging modality was employed with each technique having its distinct sensitivity and specificity for detecting cysts.

\section{Classification of Pancreatic Cystic Lesions}

3.1. Nonneoplastic Pancreatic Cysts. The most common nonneoplastic pancreatic cysts are serous cystadenomas and pancreatic pseudocysts, and these types are described in more detail in this paper. Rare nonneoplastic pancreatic cysts include true cysts, retention cysts, and lymphoepithelial cysts.

3.1.1. Serous Cystadenoma. Patients with serous cystadenomas (SCNs) are predominantly elderly women with a median age of approximately 60 years, and the cysts can arise in any region of the pancreas.

Classical features of a serous cystadenoma include microcystic morphology, a central area of calcification, and a watery, nonviscous fluid content. However a macrocystic variant of serous cystadenomas exists and can easily be confused with a pseudocyst or a mucinous cystadenoma [7-9]. Serous cystadenomas are lined by a glycogen-rich cuboidal epithelium which can be shown with cytopathological analysis [10]. Although a small number of cases of malignant serous cystadenocarcinomas have been described, it is generally believed that serous cystadenomas have virtually no malignant potential [11]. Serous cystadenomas can be treated conservatively if the patient is asymptomatic. Surgery is treatment of choice when a patient has symptoms or the distinction between a serous cystadenoma and a mucinous cystic neoplasm is not possible.

3.1.2. Pseudocysts. Pancreatic pseudocysts are fluid collections arising from leakage of the pancreatic duct lacking an epithelial lining. They usually occur following the course of an acute pancreatitis, chronic pancreatitis or secondary to an abdominal trauma [12]. The incidence of pseudocysts in the phase of an acute pancreatitis is $5.1 \%$ to $16 \%$ [13-15] whereas the incidence in chronic pancreatitis is higher with percentages varying from $20 \%$ to $40 \%$ [16-18].

Radiologic imaging of pseudocysts frequently shows a single cystic lesion, without septations or solid components. Aspirated fluid often has a low viscosity, high amylase, and cytology which is consistent with an inflammatory origin. The cysts are often filled with protease-free serous fluid if no connection to the pancreatic duct exists. Whereas size of $>6 \mathrm{~cm}$ and duration of more than 6 weeks used to be main indicators for intervention, currently symptomatology is the main indicator for intervention.

3.2. Neoplastic Pancreatic Cysts. The majority of neoplastic cysts are represented by mucinous cystic neoplasms (MCNs) (10-49\%) and intraductal papillary mucinous neoplasm (IPMN) (21-33\%) [19, 20]. Solid pseudopapillary neoplasms are less common. Other rare neoplastic cystic lesions include cystic neuroendocrine tumors and acinar cell cystadenocarcinomas but these will not be discussed in this paper.

3.2.1. Mucinous Cystic Neoplasm. Patients with MCNs are almost exclusively middle-aged women [21, 22], and most of the MCNs appear in the body or tail of the pancreas although they occasionally may occur in the head. The average size of the cysts is larger than $5 \mathrm{~cm}$ at time of presentation [2224]. MCNs are generally macrocystic, thick-walled cysts that typically lack communication with the ductal system [25, 26]. A microcystic MCN is rarely seen [27, 28]. They are either unilocular or multilocular with a small number of compartments [29]. Unique is the fact that MCNs contain a mucinous, dense ovarian stroma surrounding the epithelial cells, which is never seen in other cystic lesions. Therefore, ovariantype stroma is considered a requisite to distinguish MCNs from the other cystic neoplasms.

3.2.2. Intraductal Papillary Mucinous Neoplasms. IPMNs are slightly more often seen in male patients and they are usually 
TABLE 1: Characteristics of studies on pancreatic cyst prevalence.

\begin{tabular}{|c|c|c|c|c|}
\hline Study & $\begin{array}{c}\text { Number of } \\
\text { patients }\end{array}$ & Prevalence (\%) & Technique & $\begin{array}{c}\text { Patients with } \\
\text { known pancreatic } \\
\text { disease excluded }\end{array}$ \\
\hline de Jong et al. [1], 2010 & 2803 & 2.4 & MRI & Yes \\
\hline Laffan et al. [2], 2008 & 2832 & 2.6 & CT & Yes \\
\hline Lee et al. [3], 2010 & 616 & 13.5 & MRI & Yes \\
\hline Spinelli et al. [4], 2004 & 24039 & 1.2 & MRI and CT & No \\
\hline Zhang et al. [5], 2002 & 1444 & 19.6 & MRI & No \\
\hline Kimura et al. [6], 1995 & 300 & 24.3 & autopsy & No \\
\hline
\end{tabular}

TABLE 2: Characteristics of different pancreatic cysts.

\begin{tabular}{|c|c|c|c|c|c|}
\hline & $\mathrm{MCN}$ & IPMN & SPN & SCN & Pseudocyst \\
\hline Sex distribution & $\mathrm{F}>\mathrm{M}$ & $\mathrm{M}=\mathrm{F}$ & $\mathrm{F}>\mathrm{M}$ & $\mathrm{F}>\mathrm{M}$ & $\mathrm{F}=\mathrm{M}$ \\
\hline Age & $40-60$ & $60-70$ & $20-30$ & $60-70$ & All ages \\
\hline Average size of cyst & $>3 \mathrm{~cm}$ & $<3 \mathrm{~cm}$ & $>3 \mathrm{~cm}$ & $>3 \mathrm{~cm}$ & $>3 \mathrm{~cm}$ \\
\hline Morphologic characteristics & $\begin{array}{l}\text { Septations } \\
\text { thickened wall } \\
\text { macrocystic }\end{array}$ & $\begin{array}{l}\text { Dilatation of PD } \\
\text { micro/macrocystic }\end{array}$ & $\begin{array}{l}\text { Mixed solid and fluid } \\
\text { with hemorrhage }\end{array}$ & Microcystic & $\begin{array}{l}\text { Unilocular thick } \\
\text { wall }\end{array}$ \\
\hline Fluid & Viscous, clear & Viscous, clear & Thin, bloody & Thin, clear & Thin, dark \\
\hline Malignant potential & Yes & Yes & Yes & No & No \\
\hline
\end{tabular}

MCN: mucinous cystic neoplasm, IPMN: intraductal papillary mucinous neoplasm, SPN: solid pseudopapillary neoplasm, SCN: serous cystic neoplasm, PD: pancreatic duct.

older at presentation than patients with $\mathrm{MCNs}$ or serous cystadenomas. Most of the IPMNs arise in the head and uncinate process of the pancreas, and they are typically connected to the ductal system of the pancreas. IPMNs comprise lesions of the main pancreatic duct, side branches or a combination of these two. They have mixed features of microcystic and macrocystic lesions, and the main pancreatic duct is often dilated. IPMNs contain mucinous fluid which is sometimes extruding from the ampulla of Vater. An important difference in prevalence of malignancy exists for main-duct and side-branch IPMNs. The prevalence of malignancy for lesions of the main-duct IPMN is $57-92 \%$ whereas it is $6-46 \%$ for lesions of side-branch IPMN [30].

3.2.3. Solid Pseudopapillary Neoplasms. Solid pseudopapillary neoplasms (SPNs) are rare lesions which make up 1-2\% of all pancreatic cystic neoplasms [31,32]. They are almost exclusively found in young women with a median age of 30 years [33-35]. On the basis of the largest review [36], tumors ranged in size from 0.5 to $34.5 \mathrm{~cm}$ with a mean diameter of $6.08 \mathrm{~cm}$.

They are equally distributed throughout the pancreas [36]. Solid pseudopapillary neoplasms often start as solid tumors and undergo degeneration giving it a cystic appearance on radiologic imaging [34]. On CT and MRI, the tumor is often well circumscribed, encapsulated, and heterogeneous with hemorrhagic and cystic degeneration [32]. Solid pseudopapillary neoplasms are tumors with relatively low malignant potential, with a reported incidence of malignant transformation of $15 \%$ [34]. Surgical resection of distant metastases is justified due to the excellent long-term prognosis in the presence of metastatic disease [37]. Characteristics of different pancreatic cysts are summarized in Table 2.

\section{Clinical Presentation}

Many patients with cystic lesions of the pancreas present without abdominal complaints [38]. Lesions are often detected when a radiologic examination is performed for another reason or when an individual decides to undergo preventive screening investigations. When the pancreatic cyst is symptomatic, patients may present with epigastric pain, postprandial fullness, palpable mass, gastric outlet obstruction, nausea, vomiting, diarrhoea, steatorrhea, and/or weight loss. Patients with IPMNs sometimes present with recurrent episodes of pancreatitis. Side-branch IPMNs are more often asymptomatic than main-duct IPMNs. MCNs and pseudopapillary neoplasms are frequently large at time of diagnosis and symptoms are more common in these patients. When an advanced cystic neoplasm exists, patients often present with complaints similar to pancreatic adenocarcinoma such as pain, weight loss, and jaundice [39].

\section{Diagnostics}

Diagnostic methods that can be valuable in the differentiation of pancreatic cysts include radiologic imaging techniques such as abdominal ultrasound (US), computed tomography (CT), and magnetic resonance imaging (MRI). Endoscopic ultrasonography (EUS) and EUS-guided fine 


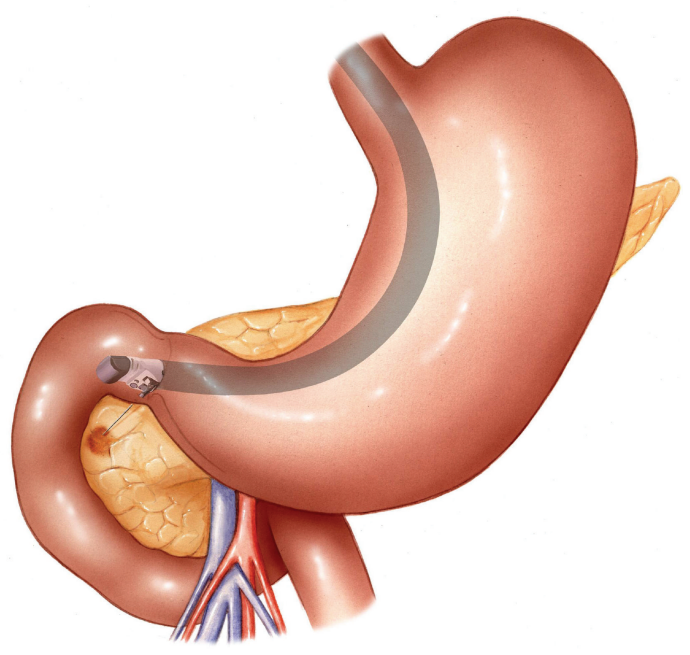

FIgURE 2: Cystic lesion in the pancreatic head is punctured using a linear array echo-endoscope.

needle aspiration (EUS-FNA) for cytopathologic examination, tumormarker determination, and molecular analysis are also widely used (Figures 2, 3, and 4).

Transabdominal ultrasonography is a safe imaging technique without radiation exposure which is helpful in the differentiation of solid and cystic lesions. It is currently widely used in the evaluation of abdominal complaints. As a result, cystic lesions are often initially detected with this modality. It is however not the imaging of first choice since it is difficult to visualize the complete pancreas due to overlying bowel or fat, and it is rather operator dependent. CT is often used in the diagnostic workup. It is a widely used imaging technique to visualize and differentiate pancreatic cysts based on morphologic features as size, microcystic/macrocystic aspect, presence of septations, nodules, and calcifications [40, 41]. MRI has the additional advantage to show a possible connection with the pancreatic duct which on T2-weighted image sequences is better visualized than with CT [42]. Another advantage of MRI, especially for follow up of the cysts, is the lack of radiation exposure.

EUS has emerged as a useful diagnostic technique in the evaluation of pancreatic cystic lesions, providing fine detail on the characteristics of the cyst because of the very high spatial resolution. It has therefore been suggested as an ideal imaging technique for pancreatic cysts [27, 4345]. EUS can image characteristics of the cysts as well as the parenchymal changes and has a role in determining the resectability if malignancy is present [46]. Despite the fact that EUS is presently widely used for the differential diagnosis, a number of points of discussion still exist. Since EUS is invasive, technically difficult, and expensive, it is not available in all hospitals. Furthermore there is a substantial interobserver agreement between endosonographers. In a multicenter study 8 experienced endosonographers reviewed videotapes of 31 EUS procedures of pancreatic cysts. In this study there was only poor to moderate agreement for the diagnosis of neoplastic versus nonneoplastic, specific type,

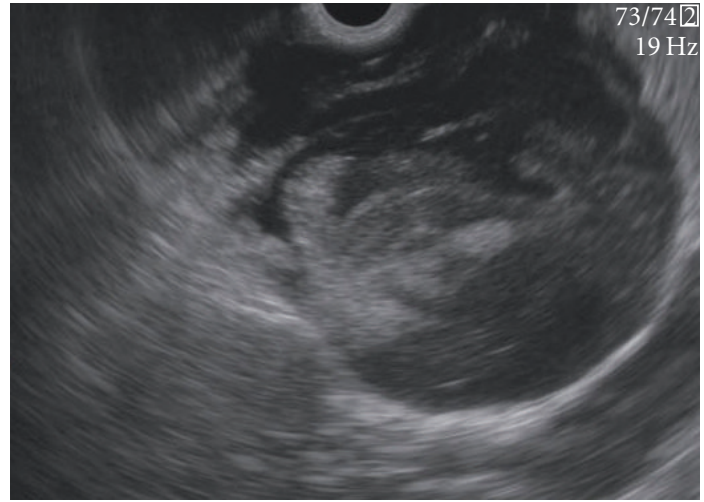

(a)

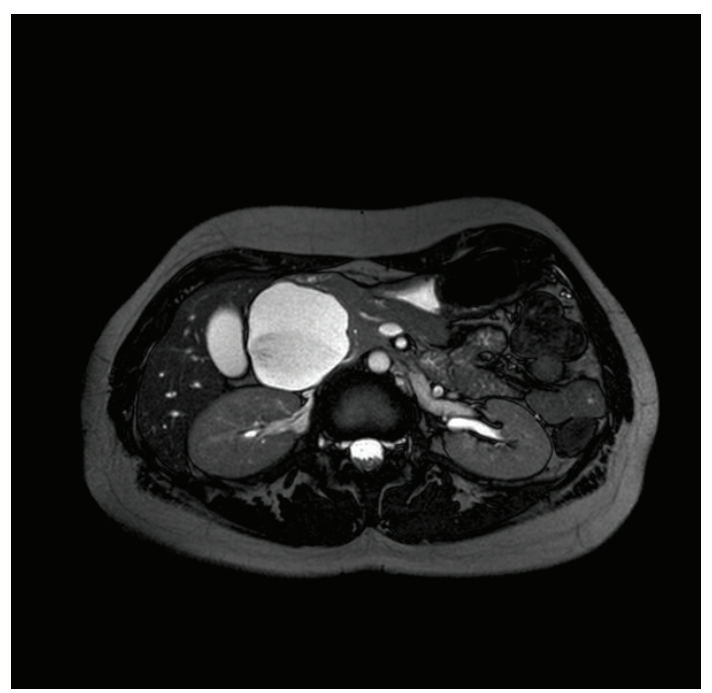

(b)

FIGURE 3: (a) EUS image of a malignant IPMN in the head of the pancreas. (b) MRI image of a malignant IPMN in the head of the pancreas.

and EUS features [47]. An advantage of EUS is the possibility to perform FNA for analysis of the cyst fluid. EUS-FNA is considered a safe technique to obtain pancreatic cyst fluid with rare, mostly mild complications, but infection, pancreatitis, and intracystic haemorrhage have been reported $[48,49]$. Infection of cysts after FNA is rare and, although common practice in most centers, data are lacking to support the use of prophylactic antibiotics. Furthermore, to minimize the risks of subsequent infection one should keep the number of punctures to a minimum and attempt to aspirate all fluid from the cyst whenever possible. Intracystic hemorrhage is a complication that occurred in $6 \%$ of all cases reported by Varadarajulu et al. but most of the complications were mild and did not need further medical intervention [50].

Cytological evaluation of pancreatic cyst fluid is widely used, and several studies report a sensitivity of approximately $50 \%$ for the differentiation of mucinous and nonmucinous pancreatic neoplasms [51-53]. However, other studies show less positive results since cytopathology is often nondiagnostic due to the low cellularity of the obtained cyst 


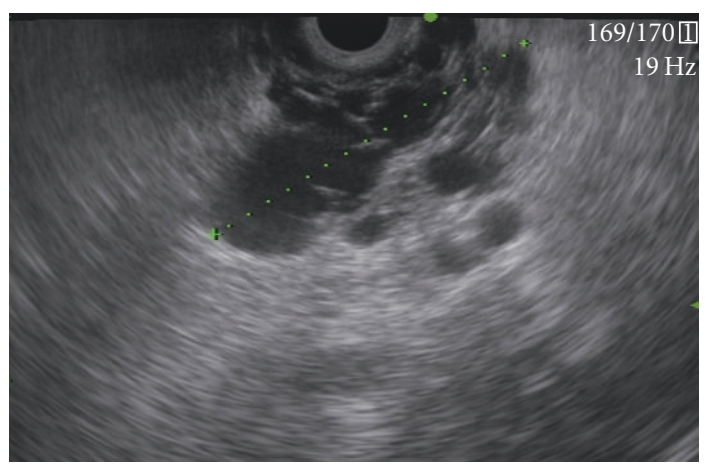

(a)

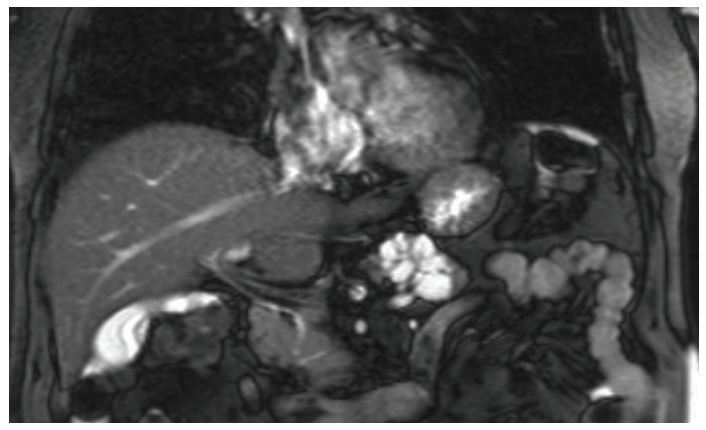

(b)

FIgURE 4: (a) EUS image of a serous cystadenoma in the head of the pancreas. (b) MRI image of a serous cystadenoma in the head of the pancreas.

fluid $[54,55]$. Biochemical analysis of cyst fluid and tumor markers have been evaluated for several years with the underlying thought that markers secreted into the cyst fluid identify the epithelial lining. Amylase is usually elevated in pseudocysts and IPMNs and low in MCNs and serous cystadenomas. Of the tumor markers, CEA is considered the best discriminant marker to differentiate between a mucinous and a nonmucinous cyst $[54,56]$. A low CEA level $(<5 \mathrm{ng} / \mathrm{mL})$ has been shown to have a sensitivity between $50 \%$ and $100 \%$ and a specificity of $77-95 \%$ to differentiate between mucinous and nonmucinous cysts [51]. Pseudocysts and serous cystadenomas generally have a low CEA value. Currently, the most widely used cutoff for an elevated CEA is $192 \mathrm{ng} / \mathrm{mL}$, which was established in a study by Brugge et al. as diagnostically sensitive in $75 \%$ and specific in $84 \%$ to discriminate between mucinous and nonmucinous cysts [54]. Altogether, the current yield of FNA is small, which can be caused by the microcystic aspect of a cyst, the high viscosity of the fluid or the minimum amount of fluid that is needed for certain examinations of the fluid. The standard use of a $19 \mathrm{G}$ needle could be helpful to aspirate both larger cysts and cysts which contain fluid with a high viscosity.

\section{Management (Guidelines)}

The most recent guideline for the management of pancreatic cyst was published in 2007 by Khalid and Brugge [57]. In this guideline the authors advice to thoroughly evaluate each incidental pancreatic cyst since many cysts are premalignant (MCN and IPMN). The initial imaging test proposed is a contrast-enhanced triphasic multidetector CT scan, which may be followed by EUS-FNA in particular cases when FNA is needed for CEA level or to puncture a solid component. Resection is recommended in all MCNs and main-duct IPMNs. Firm recommendations for the management of branch-duct IPMNs are not provided. Serous cystadenomas should only be resected if symptomatic or if the diagnosis remains in doubt. All pseudopapillary neoplasms should be considered for resection. No general guidelines are provided for the interval of follow up when surgery is not undertaken. The authors state that this decision depends on the kind of lesion and the reason why surgery was not performed.

The American Society for Gastrointestinal Endoscopy issued a guideline on the use of EUS in the management of pancreatic cysts [58]. Cystic lesions of the pancreas require diagnostic evaluation regardless of size, and EUS alone is considered not accurate enough to definitively diagnose the type of cystic lesion or to determine its malignant potential. Furthermore, FNA is advised with a low sensitivity of cytologic analysis but a high specificity for MCN and malignancies. Biochemical analysis may provide clinically useful information but cannot provide a definitive diagnosis or determine whether the lesion is malignant. In this guideline it is stated that there are currently no accepted endoscopic therapies for cystic neoplasms of the pancreas, and there is a role for endoscopic drainage of inflammatory pancreatic fluid collections.

In 2005 international consensus guidelines for the management of IPMNs and MCNs were published in which a list of clinically relevant questions and answers is provided [30]. The recommendation is to resect all main-duct and mixed variant IPMNs regardless of size as long as the patient is a good surgical candidate. Asymptomatic side-branch IPMNs can be followed with CT or MRI as long as there are no mural nodes, dilatation of the main duct or growth in size. The authors do not explicitly state that all branchduct IPMNs $>3 \mathrm{~cm}$ should be resected. More data based on branch-duct IPMNs $>3 \mathrm{~cm}$ without main-duct dilatation or mural nodules are needed to determine if all branch-duct IPMNs $>3 \mathrm{~cm}$ should be resected immediately. The authors state that MCNs should always be resected unless there are contraindications for surgery.

\section{Future Developments}

New methods to improve the yield of FNA are urgently required. Existing tumor markers have only limited value, and more sensitive biomarkers need to be identified. New techniques including proteomics and molecular analysis may be helpful for the differential diagnosis of pancreatic cysts [59].

Also the development of new techniques to minimize the fluid needed for examinations may well be useful. Furthermore, the development of new techniques to increase the cellularity of the obtained fluid could be helpful. Three reports have been recently published, studying a new type of brush 
(EchoBrush, Cook Medical) to improve the yield of cytologic examination [60-62]. These studies suggest that this relatively new technique improves the yield, but larger randomized trials are necessary to confirm these results and to define the safety profile of this more aggressive approach.

Currently, no accepted endoscopic treatment option for neoplastic cystic lesions is available but a few experimental studies have been performed to determine the safety and effectiveness of EUS-guided ethanol lavage with paclitaxel to treat pancreatic cysts [63-65]. The first studies report that this technique is a safe and feasible but larger studies with longer follow up are necessary.

\section{Conclusion}

Patients presenting with pancreatic cysts have to be thoroughly evaluated. Cross-sectional imaging should be used for the morphological characterization, and EUS-FNA for fluid and tissue sampling could be used in particular cases to discriminate between mucinous and nonmucinous cysts. Management should be based upon on carefully weighting the malignant potential of a pancreatic cystic lesions and the risk of surgery. Larger prospective studies with longer follow up are needed to increase the knowledge of the natural history of pancreatic cysts.

\section{References}

[1] K. de Jong, C. Y. Nio, J. J. Hermans et al., "High prevalence of pancreatic cysts detected by screening magnetic resonance imaging examinations," Clinical Gastroenterology and Hepatology, vol. 8, no. 9, pp. 806-811, 2010.

[2] T. A. Laffan, K. M. Horton, A. P. Klein et al., "Prevalence of unsuspected pancreatic cysts on MDCT," American Journal of Roentgenology, vol. 191, no. 3, pp. 802-807, 2008.

[3] K. S. Lee, A. Sekhar, N. M. Rofsky, and I. Pedrosa, "Prevalence of incidental pancreatic cysts in the adult population on MR imaging," American Journal of Gastroenterology, vol. 105, no. 9, pp. 2079-2084, 2010.

[4] K. S. Spinelli, T. E. Fromwiller, R. A. Daniel et al., "Cystic pancreatic neoplasms: observe or operate," Annals of Surgery, vol. 239, no. 5, pp. 651-659, 2004.

[5] X. M. Zhang, D. G. Mitchell, M. Dohke, G. A. Holland, and L. Parker, "Pancreatic cysts: depiction on single-shot fast spinecho MR images," Radiology, vol. 223, no. 2, pp. 547-553, 2002.

[6] W. Kimura, H. Nagai, A. Kuroda, T. Muto, and Y. Esaki, "Analysis of small cystic lesions of the pancreas," International Journal of Pancreatology, vol. 18, no. 3, pp. 197-206, 1995.

[7] D. Chatelain, P. Hammel, D. O'Toole et al., "Macrocystic form of serous pancreatic cystadenoma," American Journal of Gastroenterology, vol. 97, no. 10, pp. 2566-2571, 2002.

[8] B. Khurana, K. J. Mortele, J. Glickman, S. G. Silverman, and P. R. Ros, "Macrocystic serous adenoma of the pancreas: radiologic-pathologic correlation," American Journal of Roentgenology, vol. 181, no. 1, pp. 119-123, 2003.

[9] K. Lewandrowski, A. Warshaw, and C. Compton, "Macrocystic serous cystadenoma of the pancreas: a morphologic variant differing from microcystic adenoma," Human Pathology, vol. 23, no. 8, pp. 871-875, 1992.
[10] J. L. Frossard, P. Amouyal, G. Amouyal et al., "Performance of endosonography-guided fine needle aspiration and biopsy in the diagnosis of pancreatic cystic lesions," American Journal of Gastroenterology, vol. 98, no. 7, pp. 1516-1524, 2003.

[11] T. Matsumoto, S. Hirano, K. Yada et al., "Malignant serous cystic neoplasm of the pancreas: report of a case and review of the literature," Journal of Clinical Gastroenterology, vol. 39, no. 3, pp. 253-256, 2005.

[12] A. L. Warshaw, "Pancreatic cysts and pseudocysts: new rules for a new game," British Journal of Surgery, vol. 76, no. 6, pp. 533-534, 1989.

[13] E. L. Bradley, A. C. Gonzalez, and J. L. Clements Jr., "Acute pancreatic pseudocysts: incidence and implications," Annals of Surgery, vol. 184, no. 6, pp. 734-737, 1976.

[14] A. Maringhini, G. Uomo, R. Patti et al., "Pseudocysts in acute nonalcoholic pancreatitis: incidence and natural history," Digestive Diseases and Sciences, vol. 44, no. 8, pp. 1669-1673, 1999.

[15] N. J. London, J. P. Neoptolemos, J. Lavelle, I. Bailey, and D. James, "Serial computed tomography scanning in acute pancreatitis: a prospective study," Gut, vol. 30, no. 3, pp. 397-403, 1989.

[16] M. Barthet, M. Bugallo, L. S. Moreira, C. Bastid, B. Sastre, and J. Sahel, "Management of cysts and pseudocysts complicating chronic pancreatitis. A retrospective study of 143 patients," Gastroenterologie Clinique et Biologique, vol. 17, no. 4, pp. 270276, 1993.

[17] R. W. Ammann, A. Akovbiantz, F. Largiader, and G. Schueler, "Course and outcome of chronic pancreatitis. Longitudinal study of a mixed medical-surgical series of 245 patients," Gastroenterology, vol. 86, no. 5, pp. 820-828, 1984.

[18] D. W. Elliott, "Pancreatic pseudocysts," Surgical Clinics of North America, vol. 55, no. 3, pp. 339-362, 1975.

[19] C. C. Fernandez-del Castillo, J. Targarona, S. P. Thayer, D. W. Rattner, W. R. Brugge, and A. L. Warshaw, "Incidental pancreatic cysts: clinicopathologic characteristics and comparison with symptomatic patients," Archives of Surgery, vol. 138, no. 4, pp. 427-434, 2003.

[20] N. V. Adsay, D. S. Klimstra, and C. C. Compton, "Cystic lesions of the pancreas. Introduction," Seminars in Diagnostic Pathology, vol. 17, no. 1, pp. 1-6, 2000.

[21] M. G. Sarr, H. A. Carpenter, L. P. Prabhakar et al., "Clinical and pathologic correlation of 84 mucinous cystic neoplasms of the pancreas: can one reliably differentiate benign from malignant (or premalignant) neoplasms?" Annals of Surgery, vol. 231, no. 2, pp. 205-212, 2000.

[22] S. Crippa, R. Salvia, A. L. Warshaw et al., "Mucinous cystic neoplasm of the pancreas is not an aggressive entity: lessons from 163 resected patients," Annals of Surgery, vol. 247, no. 4, pp. 571-579, 2008.

[23] G. H. Sakorafas and M. G. Sarr, "Cystic neoplasms of the pancreas; what a clinician should know," Cancer Treatment Reviews, vol. 31, no. 7, pp. 507-535, 2005.

[24] A. L. Mulkeen, P. S. Yoo, and C. Cha, "Less common neoplasms of the pancreas," World Journal of Gastroenterology, vol. 12, no. 20, pp. 3180-3185, 2006.

[25] R. Salvia, L. Festa, G. Butturini et al., "Pancreatic cystic tumors," Minerva Chirurgica, vol. 59, no. 2, pp. 185-207, 2004.

[26] F. Campbell and B. Azadeh, "Cystic neoplasms of the exocrine pancreas," Histopathology, vol. 52, no. 5, pp. 539-551, 2008.

[27] W. R. Brugge, "The role of EUS in the diagnosis of cystic lesions of the pancreas," Gastrointestinal Endoscopy, vol. 52, supplement 6, pp. S18-S22, 2000. 
[28] W. R. Brugge, "Evaluation of pancreatic cystic lesions with EUS," Gastrointestinal Endoscopy, vol. 59, no. 6, pp. 698-707, 2004.

[29] N. C. Balci and R. C. Semelka, "Radiologic features of cystic, endocrine and other pancreatic neoplasms," European Journal of Radiology, vol. 38, no. 2, pp. 113-119, 2001.

[30] M. Tanaka, S. Chari, V. Adsay et al., "International consensus guidelines for management of intraductal papillary mucinous neoplasms and mucinous cystic neoplasms of the pancreas," Pancreatology, vol. 6, no. 1-2, pp. 17-32, 2006.

[31] R. C. Martin, D. S. Klimstra, M. F. Brennan, and K. C. Conlon, "Solid-pseudopapillary tumor of the pancreas: a surgical enigma?” Annals of Surgical Oncology, vol. 9, no. 1, pp. 35-40, 2002.

[32] D. S. Klimstra, B. M. Wenig, and C. S. Heffess, "Solid-pseudopapillary tumor of the pancreas: a typically cystic carcinoma of low malignant potential," Seminars in Diagnostic Pathology, vol. 17, no. 1, pp. 66-80, 2000.

[33] C. Mao, M. Guvendi, D. R. Domenico, K. Kim, N. R. Thomford, and J. M. Howard, "Papillary cystic and solid tumors of the pancreas: a pancreatic embryonic tumor? Studies of three cases and cumulative review of the world's literature," Surgery, vol. 118, no. 5, pp. 821-828, 1995.

[34] S. G. Tipton, T. C. Smyrk, M. G. Sarr, and G. B. Thompson, "Malignant potential of solid pseudopapillary neoplasm of the pancreas," British Journal of Surgery, vol. 93, no. 6, pp. 733737, 2006

[35] E. Panieri, J. E. Krige, P. C. Bornman, S. M. Graham, J. Terblanche, and J. P. Cruse, "Operative management of papillary cystic neoplasms of the pancreas," Journal of the American College of Surgeons, vol. 186, no. 3, pp. 319-324, 1998.

[36] T. Papavramidis and S. Papavramidis, "Solid pseudopapillary tumors of the pancreas: review of 718 patients reported in english literature," Journal of the American College of Surgeons, vol. 200, no. 6, pp. 965-972, 2005.

[37] S. M. de Castro, D. Singhal, D. C. Aronson et al., "Management of solid-pseudopapillary neoplasms of the pancreas: a comparison with standard pancreatic neoplasms.", World Journal of Surgery, vol. 31, no. 5, pp. 1130-1135, 2007.

[38] C. Bassi, R. Salvia, E. Molinari, C. Biasutti, M. Falconi, and P. Pederzoli, "Management of 100 consecutive cases of pancreatic serous cystadenoma: wait for symptoms and see at imaging or vice versa?" World Journal of Surgery, vol. 27, no. 3, pp. 319323, 2003.

[39] D. L. Kerlin, C. F. Frey, B. I. Bodai, P. L. Twomey, and B. Ruebner, "Cystic neoplasms of the pancreas," Surgery Gynecology and Obstetrics, vol. 165, no. 6, pp. 475-478, 1987.

[40] C. A. Curry, J. Eng, K. M. Horton et al., "CT of primary cystic pancreatic neoplasms: can CT be used for patient triage and treatment?" American Journal of Roentgenology, vol. 175, no. 1, pp. 99-103, 2000.

[41] M. Minami, Y. Itai, K. Ohtomo, H. Yoshida, K. Yoshikawa, and M. Iio, "Cystic neoplasms of the pancreas: comparison of MR imaging with CT," Radiology, vol. 171, no. 1, pp. 53-56, 1989.

[42] K. Koito, T. Namieno, T. Ichimura et al., "Mucin-producing pancreatic tumors: comparison of MR cholangiopancreatography with endoscopic retrograde cholangiopancreatography," Radiology, vol. 208, no. 1, pp. 231-237, 1998.

[43] J. F. Tseng, A. L. Warshaw, D. V. Sahani et al., "Serous cystadenoma of the pancreas: tumor growth rates and recommendations for treatment," Annals of Surgery, vol. 242, no. 3, pp. 413-421, 2005.
[44] K. Yamaguchi and M. Tanaka, "Radiologic imagings of cystic neoplasms of the pancreas," Pancreatology, vol. 1, no. 6, pp. 633-636, 2001.

[45] J. Ariyama, M. Suyama, K. Satoh, and K. Wakabayashi, "Endoscopic ultrasound and intraductal ultrasound in the diagnosis of small pancreatic tumors," Abdominal Imaging, vol. 23, no. 4, pp. 380-386, 1998.

[46] S. L. Brandwein, J. J. Farrell, B. A. Centeno, and W. R. Brugge, "Detection and tumor staging of malignancy in cystic, intraductal, and solid tumors of the pancreas by EUS," Gastrointestinal Endoscopy, vol. 53, no. 7, pp. 722-727, 2001.

[47] N. A. Ahmad, M. L. Kochman, C. Brensinger et al., "Interobserver agreement among endosonographers for the diagnosis of neoplastic versus non-neoplastic pancreatic cystic lesions," Gastrointestinal Endoscopy, vol. 58, no. 1, pp. 59-64, 2003.

[48] L. S. Lee, J. R. Saltzman, B. C. Bounds, J. M. Poneros, W. R. Brugge, and C. C. Thompson, "EUS-guided fine needle aspiration of pancreatic cysts: a retrospective analysis of complications and their predictors," Clinical Gastroenterology and Hepatology, vol. 3, no. 3, pp. 231-236, 2005.

[49] D. O’Toole, L. Palazzo, R. Arotcarena et al., "Assessment of complications of EUS-guided fine-needle aspiration," Gastrointestinal Endoscopy, vol. 53, no. 4, pp. 470-474, 2001.

[50] S. Varadarajulu and M. A. Eloubeidi, "Frequency and significance of acute intracystic hemorrhage during EUS-FNA of cystic lesions of the pancreas," Gastrointestinal Endoscopy, vol. 60, no. 4, pp. 631-635, 2004.

[51] L. A. van der Waaij, H. M. van Dullemen, and R. J. Porte, "Cyst fluid analysis in the differential diagnosis of pancreatic cystic lesions: a pooled analysis," Gastrointestinal Endoscopy, vol. 62, no. 3, pp. 383-389, 2005.

[52] R. M. Walsh, J. M. Henderson, D. P. Vogt et al., "Prospective preoperative determination of mucinous pancreatic cystic neoplasms," Surgery, vol. 132, no. 4, pp. 628-634, 2002.

[53] H. C. Oh, M. H. Kim, C. Y. Hwang et al., "Cystic lesions of the pancreas: challenging issues in clinical practice," American Journal of Gastroenterology, vol. 103, no. 1, pp. 229-239, 2008.

[54] W. R. Brugge, K. Lewandrowski, E. Lee-Lewandrowski et al., "Diagnosis of pancreatic cystic neoplasms: a report of the cooperative pancreatic cyst study," Gastroenterology, vol. 126, no. 5, pp. 1330-1336, 2004.

[55] B. A. Centeno, A. L. Warshaw, W. Mayo-Smith, J. F. Southern, and K. Lewandrowski, "Cytologic diagnosis of pancreatic cystic lesions: a prospective study of 28 percutaneous aspirates," Acta Cytologica, vol. 41, no. 4, pp. 972-980, 1997.

[56] K. B. Lewandrowski, J. F. Southern, M. R. Pins, C. C. Compton, and A. L. Warshaw, "Cyst fluid analysis in the differential diagnosis of pancreatic cysts: a comparison of pseudocysts, serous cystadenomas, mucinous cystic neoplasms, and mucinous cystadenocarcinoma," Annals of Surgery, vol. 217, no. 1, pp. 41-47, 1993.

[57] A. Khalid and W. Brugge, "ACG practice guidelines for the diagnosis and management of neoplastic pancreatic cysts," American Journal of Gastroenterology, vol. 102, no. 10, pp. 2339-2349, 2007.

[58] B. C. Jacobson, T. H. Baron, D. G. Adler et al., "ASGE guideline: the role of endoscopy in the diagnosis and the management of cystic lesions and inflammatory fluid collections of the pancreas," Gastrointestinal Endoscopy, vol. 61, no. 3, pp. 363-370, 2005.

[59] E. Ke, B. B. Patel, T. Liu et al., "Proteomic analyses of pancreatic cyst fluids," Pancreas, vol. 38, no. 2, pp. e33-e42, 2009.

[60] M. Al-Haddad, M. Raimondo, T. Woodward et al., "Safety and efficacy of cytology brushings versus standard FNA in 
evaluating cystic lesions of the pancreas: a pilot study," Gastrointestinal Endoscopy, vol. 65, no. 6, pp. 894-898, 2007.

[61] M. Al-Haddad, K. R. Gill, M. Raimondo et al., "Safety and efficacy of cytology brushings versus standard fine-needle aspiration in evaluating cystic pancreatic lesions: a controlled study," Endoscopy, vol. 42, no. 2, pp. 127-132, 2010.

[62] M. Bruno, M. Bosco, P. Carucci et al., "Preliminary experience with a new cytology brush in EUS-guided FNA," Gastrointestinal Endoscopy, vol. 70, no. 6, pp. 1220-1224, 2009.

[63] H. C. Oh, D. W. Seo, T. J. Song et al., "Endoscopic ultrasonography-guided ethanol lavage with paclitaxel injection treats patients with pancreatic cysts," Gastroenterology, vol. 140, no. 1, pp. 172-179, 2011.

[64] J. DeWitt, K. McGreevy, C. M. Schmidt, and W. R. Brugge, "EUS-guided ethanol versus saline solution lavage for pancreatic cysts: a randomized, double-blind study," Gastrointestinal Endoscopy, vol. 70, no. 4, pp. 710-723, 2009.

[65] S. I. Gan, C. C. Thompson, G. Y. Lauwers, B. C. Bounds, and W. R. Brugge, "Ethanol lavage of pancreatic cystic lesions: initial pilot study," Gastrointestinal Endoscopy, vol. 61, no. 6, pp. 746-752, 2005. 


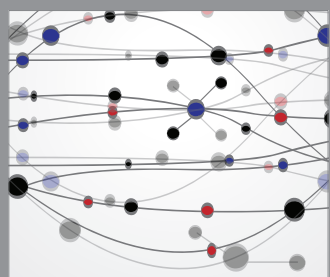

The Scientific World Journal
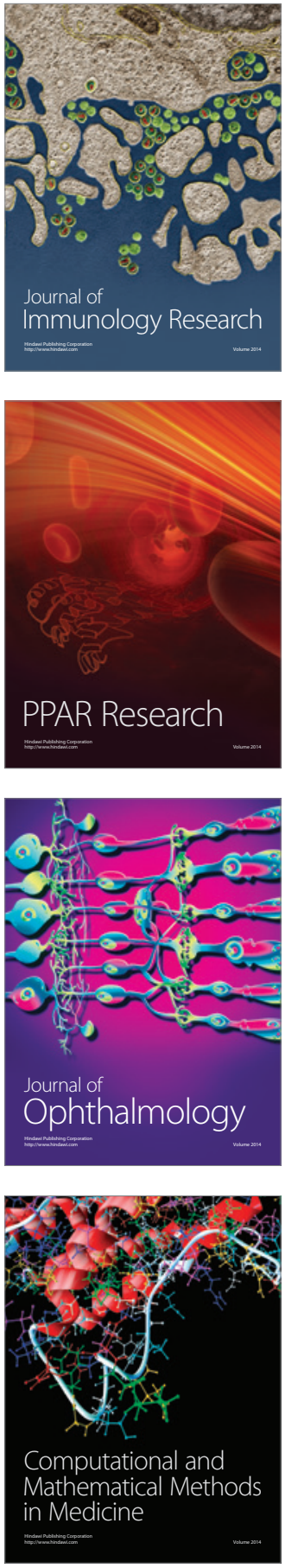

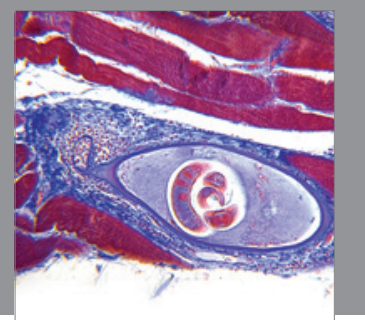

Gastroenterology

Research and Practice
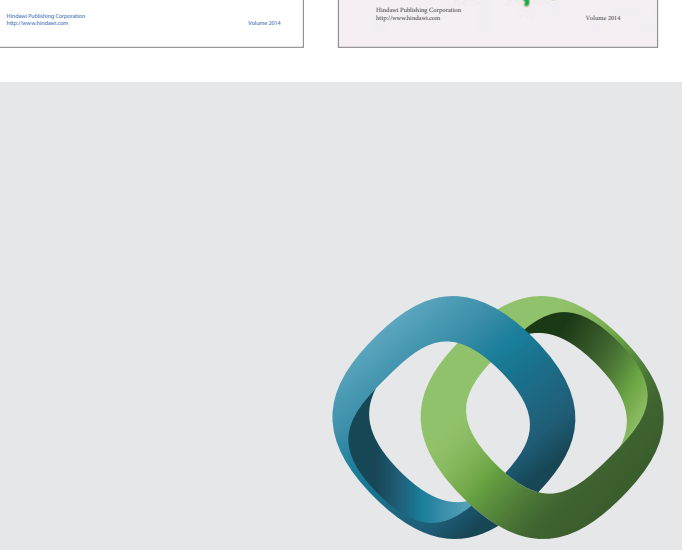

\section{Hindawi}

Submit your manuscripts at

http://www.hindawi.com
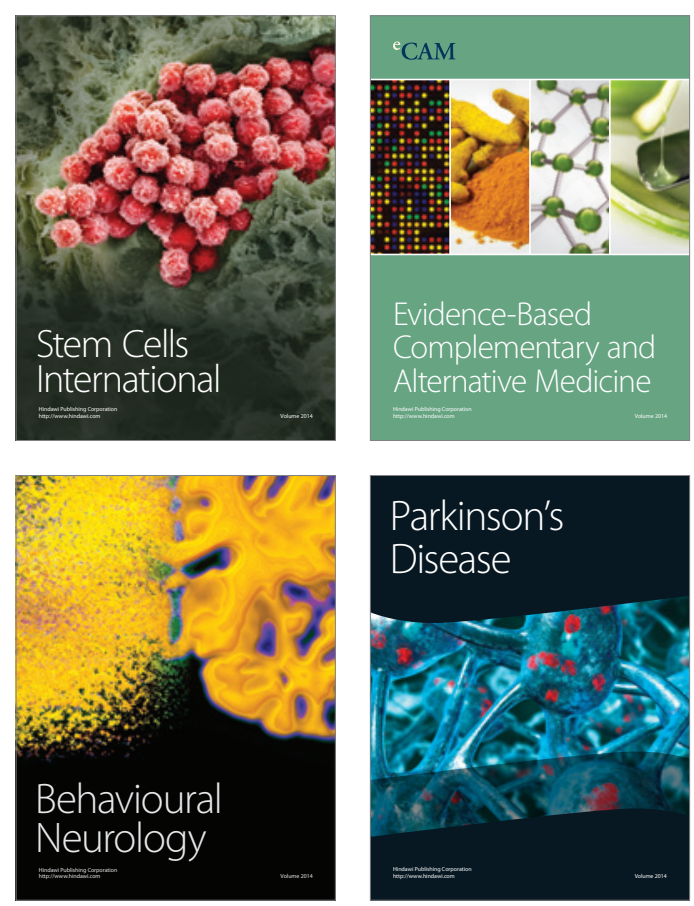

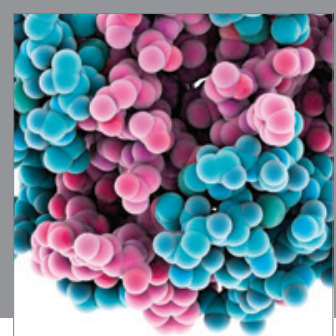

Journal of
Diabetes Research

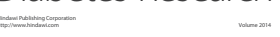

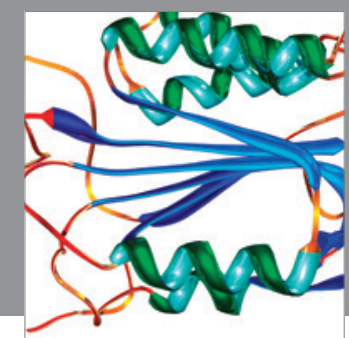

Disease Markers
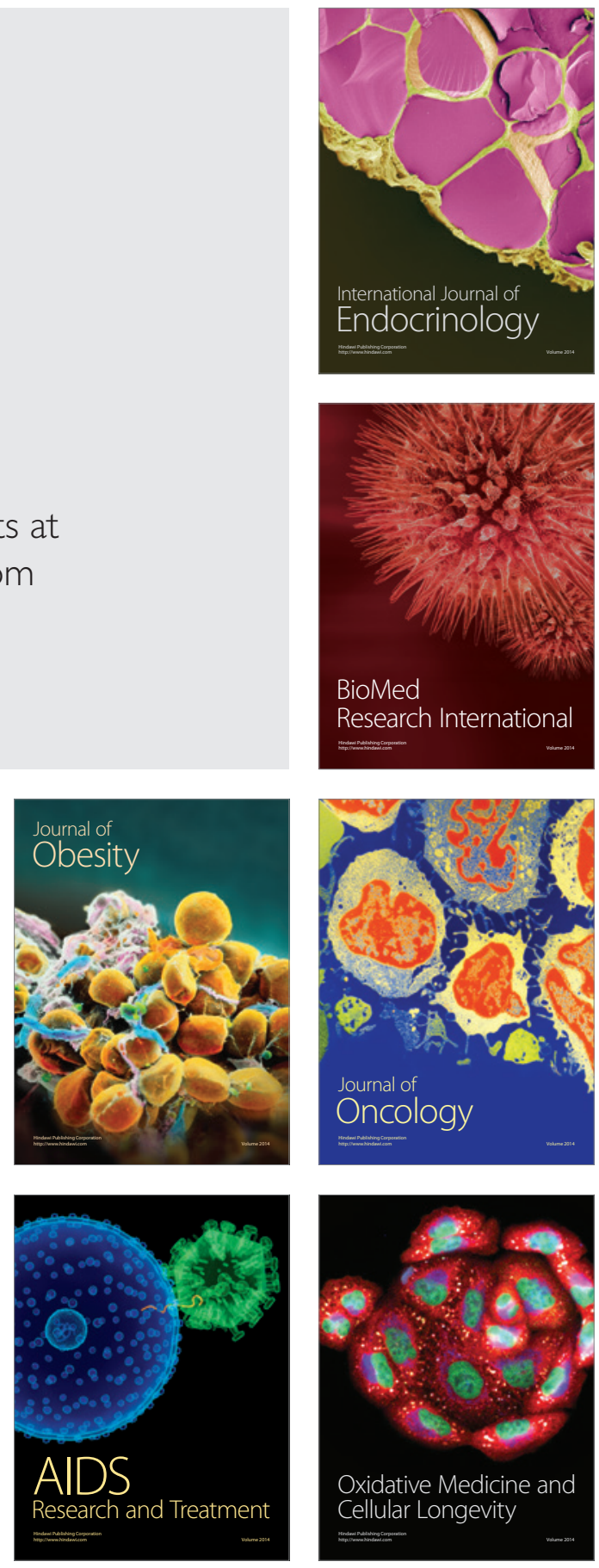